\title{
Systematic Characterization of Reciprocal Space by SAED: Advantages of a Double-Tilt, Rotate Holder
}

\section{S. Turner}

Surface and Microanalysis Science Division, Chemical Science and Technology Laboratory, National Institute of Standards and Technology, Gaithersburg, MD 20899-8371

Characterization of reciprocal space by selected area electron diffraction (SAED) has been limited by the conventional transmission electron microscope (TEM) sample holders available for sample manipulation. Traditional sample holders include the double-tilt holder and rotate-tilt holder, each of which has two degrees of freedom for sample manipulation. These holders allow for orientation of samples down zone axes. However, the microscopist commonly cannot easily tilt around a crystallographic direction of interest within the resultant electron diffraction patterns unless the sample is fortuitously oriented in the sample holder. Multiple diffraction patterns, however, can be collected from the same sample. This is done, especiallyfor higher symmetry metallurgic samples, by combining the available tilt or rotate-tilt capabilities [1]. The angles between zones can be only estimated. Alternatively, software available for the FEI CompuStage allows for tilt around a specified crystallographic direction (FEI CM k-Space Control) [2,3]. The capability is limited to those instruments with a CompuStage. In this work, the applicability of a double-tilt, rotate (2TR) holder to characterization of reciprocal space is tested. This holder has a third degree of freedom for sample manipulation and it was expected that this would allow for systematic characterization of reciprocal space by SAED.

The 2TR holder used is Gatan Model 925. It was used in an FEI CM30 in which tilt along the rod axis ( $\mathrm{x}$ axis) is limited to $\pm 45^{\circ}$ in the Twin objective lens configuration. Tilt perpendicular to the rod axis (y axis) is limited to $\pm 24^{\circ}$ by the holder configuration. A rutile particle approximately $500 \mathrm{~nm}$ long and $200 \mathrm{~nm}$ in width was studied. An SAED patern oriented down [110] was obtained using the double-tilt capabilities of the holder. The $\underline{c}$ axis of the crystallite was then aligned with the projection of the sample holder rod axis by the rotation capability of the holder (Fig. 1a). The sample holder was tilted around the $\mathrm{x}$ axis of the sample holder ([001]* of the crystallite) to obtain several SAED patterns that all included [001]*. The angles between the zones correspond simply to the difference in tilt of the $\mathrm{x}$ axis and the angles obtained were within $1^{\circ}$ of those expected (Table 1). The sample was then returned to the [110] orientation and the sample rotated so that the [-110]* was aligned with the $\mathrm{x}$ axis of the sample holder (Fig. 1b). The sample was tilted around $[-110]^{*}$ and several patterns containing [-110]* were obtained. Interzone angles could again be directly determined from the tilt of the $\mathrm{x}$ axis and were consistent with those expected. Finally, the sample was tilted to the [111] orientation and the sample tilted around the [112]* direction in this diffraction pattern. Again, several diffraction patterns containing this crystallographic direction were obtained and the measured interzone angles were consistent with those calculated.

This work confirms that it is possible to systematically examine reciprocal space by SAED using the double-tilt, rotate holder by aligning crystallographic directions of interest parallel to holder axes. It is then possible to directly obtain interzone angles, with an accuracy within approximately $1^{\circ}$ of the calculated value. It is expected that the capability of this holder for 
systematic manipulation of samples will have implications for improved phase identification and for reciprocal space mapping of complex diffraction effects.

\section{References}

[1] D.B. Williams and C.B. Carter, Transmission Electron Microscopy, Diffraction II, Plenum Press, New York (1996) and references therein.

[2] WWw.feic.com/support/cmindex.htm

[3] Certain commercial equipment, instruments, or materials are identified in thispaper to specify adequately the experimental procedure. Such identification does not imply recommendation or endorsement by the National Institute of Standards and Technology, nor does it imply that the materials or equipment are necessarily the best avalable for the purpose.
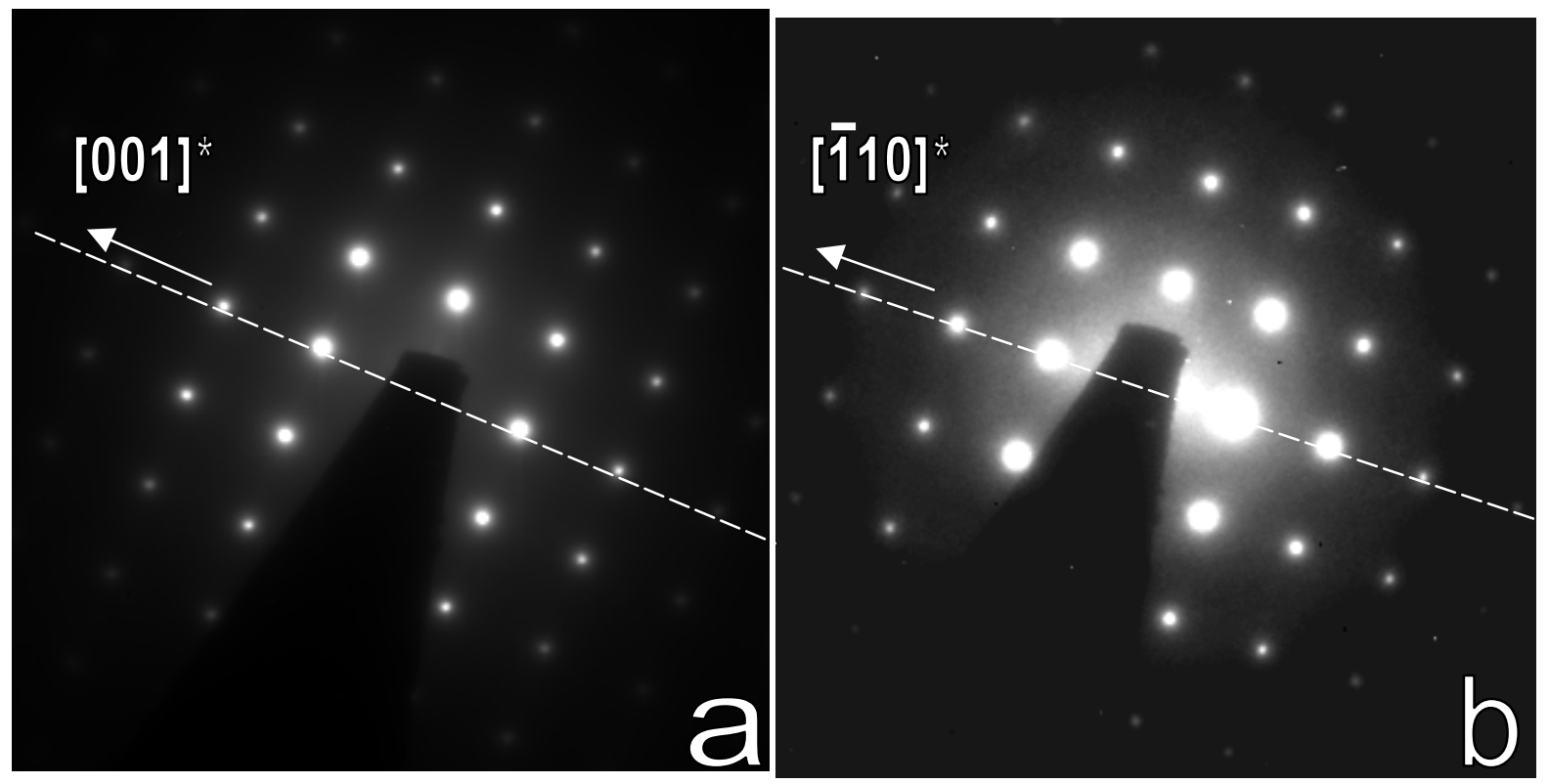

Figure 1(a) [110] SAED pattern oriented so that [001]* is parallel to the $\mathrm{x}$ axis of the sample holder, (b) [110] SAED pattern oriented so that the [-110]* is parallel to the $\mathrm{x}$ axis of the sample holder (dashed line represents projection of holder $\mathrm{x}$ axis).

Table 1. Calculated and measured interzone angles between [110] and zones obtained by tilting around [001]

\begin{tabular}{|c|c|c|}
\hline \multirow{2}{*}{ zones } & \multicolumn{2}{|c|}{ Angle between [110] and zone } \\
\cline { 2 - 3 } & Calculated & Measured \\
\hline$[140]$ & 31.0 & 31.5 \\
\hline$[120]$ & 18.4 & 19.0 \\
\hline$[110]$ & $(0)$ & $(0)$ \\
\hline$[210]$ & 18.4 & 18.5 \\
\hline$[410]$ & 31.0 & 30.0 \\
\hline$[100]$ & 45.0 & 45.0 \\
\hline
\end{tabular}

